\title{
LCF on turbogenerator rotors and coil retaining rings: material characterization and sensitivity analyses
}

\author{
G. Olmi ${ }^{1, a}$ and A. Freddi ${ }^{1}$ \\ ${ }^{1}$ DIEM Department, University of Bologna, Viale del Risorgimento 2, 40136 Bologna, Italy
}

\begin{abstract}
Turbogenerator rotors and coil retaining rings (CRRs) are highly loaded components typically subjected to LCF at any machine switch-on and switch-off. The present study aims at LCF characterization of two widely applied steels, $26 \mathrm{NiCrMoV}$ 145 (for rotor manufacturing) and $18 \mathrm{Mn} 18 \mathrm{Cr}$ (for CRR). Material anisotropy is also considered by performing an extended experimental campaign on specimens machined along different (tangential and radial) directions from trial components. The experimental tests, carried out with the use of a novel testing-constraining device for misalignment auto-compensation and with an original methodology for strain controlling, led to the determination of static, cyclic and fatigue curves for all the investigated cases. The research was completed by sensitivity analyses on the adopted models, thus determining fatigue curve tolerance bands, and by a statistical Analysis of Variance to compare the LCF performance of the different materials along the two considered machining directions. Results showed a significantly better performance of $18 \mathrm{Mn} 18 \mathrm{Cr}$ and a weak anisotropy effect, remarkable just at the highest strain values, on a reduced portion of the LCF life range.
\end{abstract}

\section{Introduction}

A turbogenerator rotor contains uniformly spaced longitudinal slots, where copper conductors are packed, held in place by metal wedges. At the end of the slots the conductors emerge to form a coil that is wound around the rotor. As the rotor spins, the copper conductors are subjected to a great centrifugal force and must be restrained. Along the rotor, this resistance is provided by the wedges, while, at the ends, coil retaining rings (CRRs) are shrunk fitted onto the body over the coils. A turbogenerator is usually designed for working at the nominal rotational speed $(3,000 \mathrm{rpm})$ of the rotor during peak hours, with stops as the energy request drops. It implies that over the whole life, about 50 years, such machines experience many transitories, from 10,000 to 15,000 (considering 200-300 per year). Both the rotor and the CRRs are subjected to fluctuating loads generating low cycle fatigue (LCF): during stops the stresses due to shrink-fit are maximum, while at the nominal rotational speed the centrifugal force tends to release such stresses, in the meantime additional stresses are generated on the CRR to react against the centrifugal force on copper coils.

The materials for CRR manufacturing must be non magnetic, with high thermal and electrical conductivity and a high coefficient of thermal expansion. From the mechanical point of view, CRR

\footnotetext{
a e-mail : giorgio.olmi@unibo.it
}

This is an Open Access article distributed under the terms of the Creative Commons Attribution-Noncommercial License 3.0, which permits unrestricted use, distribution, and reproduction in any noncommercial medium, provided the original work is properly cited. 
steels have high static and fatigue resistances, high corrosion resistance and a great fracture toughness [1].

For the aforementioned properties the $18 \mathrm{Mn} 18 \mathrm{Cr}$ steel is nowadays a widely used material for CRR manufacturing with several applicative examples in [2-6]. Steels for rotor construction are usually low-alloyed ones: they also have good fracture toughness and static and dynamic resistances, but lower than those of CRR steels. In [7] the structural analysis and the experimentation of a flywheel connected to a turbogenerator are reported, while many details on rotor design, manufacturing and testing are contained in [8-12]. The most used steel is 26 NiCrMoV 145 (3.5NiCrMoV or ASTM A470), satisfying all the aforementioned requirements.

The contribution [4] deals with an investigation on the anisotropy of such materials, with specific reference to those for CRR manufacturing. It is pointed out that $18 \mathrm{Mn} 18 \mathrm{Cr}$ steel is affected by an anisotropic behaviour. However, probably due to the influence of Nitrogen (about $0.75 \%$ concentration) such effect is lower than for other CRR materials used in the past, such as $18 \mathrm{Mn} 5 \mathrm{Cr}$. Further experimentation was performed on specimens machined from a trial CRR, investigating both the static and the fatigue performance along the radial and the tangential directions. The results showed that the fatigue strength is slightly greater in the tangential direction, relating such finding to the better yield resistance. The problem of anisotropy is investigated also in [13], with reference to fracture toughness properties.

Despite the typical LCF load, the structural analyses are not usually performed with the application of the models available in this field, such as the Manson-Coffin model. The reason is often related to the lack of information on the curves and related parameters leading the cyclic and fatigue behaviour of such steels. Many studies are sometimes performed with the same experimental settings of the high cycle fatigue. For example, the investigations on anisotropy described in [4] involved fatigue tests and the determination of the sloping part of a fatigue curve up to $10^{5}$ cycles. However, these tests and related, previously cited, results refer to the material behaviour in stress controlled conditions and not in strain controlled ones, as required for locally variable states of strain. A serious problem, which probably prevented the execution of tests in strain controlled condition is the reduced thickness of the CRR, implying a short length for the specimens machined in the radial direction. This short length is often incompatible with the application of commonly used axial extensometers. Again, regarding the anisotropy experimental studies, none regarded the rotor materials, despite the very serious consequences in the case of rotor explosion [14-16].

On the basis of the previous observation the object of the present paper was to experimentally determine the main LCF parameters, with reference to two widely used steels, for rotor and CRR manufacturing. Sensitivity and statistical analyses were performed to discuss on the reliability of the regression lines adopted for the determination of the Manson-Coffin curve and to investigate on the anisotropy of the two materials under static and fatigue load in the typical life range of LCF.

\section{Materials and Methods}

The research involved the two aforementioned materials (nominal compositions in Table 1), 26 $\mathrm{NiCrMoV} 145$ for the rotor and $18 \mathrm{Mn} 18 \mathrm{Cr}$ for the CRR. The second factor to be studied was specimen orientation (tangential or radial direction) with respect to the component layout. The experimental campaign was arranged in the two-factor plane in Table 2. For each of the four cases 40 specimens were machined for material characterization both under static and cyclic loads. The number of 40 appeared suitable, also according to previous studies in the field of LCF for turbogenerator material characterization [17]. All the specimens along the tangential direction were manufactured according to ASTM E606-04 [18]: they have a uniform gage test section and are shown in Figure 1 (A). In the case of the radial direction, CRR reduced thickness prevented from using specimens of the same type, forcing to use shorter specimens with an hourglass geometry. They have the same nominal section and are shown in Figure 2 (B). Specimens of the same type were also machined in the rotor material along the same radial direction: in this case the larger rotor 
section would have not prevented from using conformal specimens, but is was preferred to maintain the same geometry for result comparability reasons.

Table 1. Chemical compositions of the two materials.

\begin{tabular}{|c|c|c|c|c|c|}
\hline \multirow{4}{*}{$\begin{array}{l}\text { 26NiCrMoV14 } 5 \\
\text { (rotor) }\end{array}$} & $\mathrm{C}[\%]$ & $\mathrm{Cr}[\%]$ & $\mathrm{Ni}[\%]$ & Mo [\%] & $\mathrm{V}[\%]$ \\
\hline & $0,22 \div 0,32$ & $1,2 \div 1,4$ & $3,4 \div 4$ & $0,25 \div 0,45$ & $0,05 \div 0,15$ \\
\hline & $\mathrm{Mn}[\%]$ & $\mathrm{Si}[\%]$ & $\mathrm{P}[\%]$ & $\mathrm{S}[\%]$ & $\mathrm{N}[\%]$ \\
\hline & $0,15 \div 0,40$ & $<0,30$ & $<0,015$ & $<0,018$ & --- \\
\hline \multirow{4}{*}{$\begin{array}{l}18 \mathrm{Mn} 18 \mathrm{Cr} \\
(\mathrm{CRR})\end{array}$} & $\mathrm{C}[\%]$ & $\mathrm{Cr}[\%]$ & $\mathrm{Ni}[\%]$ & Мo [\%] & V [\%] \\
\hline & $\leq 0,13$ & $17,5 \div 20$ & - & - & - \\
\hline & $\mathrm{Mn}[\%]$ & Si [\%] & $\mathrm{P}[\%]$ & S [\%] & $\mathrm{N}[\%]$ \\
\hline & $17,5 \div 20,0$ & $\leq 0,60$ & 0,033 & 0,001 & $0,73 \div 0,78$ \\
\hline
\end{tabular}

Table 2. Two-factor experimental planning.

\begin{tabular}{|c|c|c|c|}
\hline & \multicolumn{2}{|c|}{ Material } \\
\hline & & 26 NiCrMoV 145 (rotor) & 18Mn18Cr (CRR) \\
\hline \multirow{2}{*}{ Direction } & tangential & 40 specimens & 40 specimens \\
\hline & radial & 40 specimens & 40 specimens \\
\hline
\end{tabular}

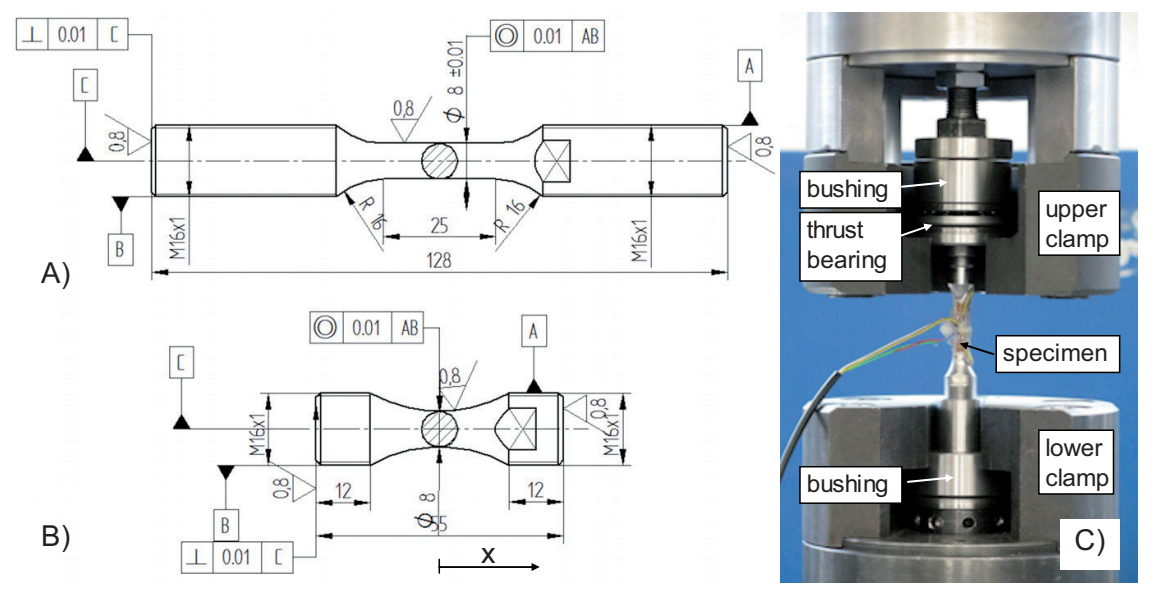

Fig. 1. Drawings of conformal (A) and shorter (B) specimens, loading-constraining device (C).

All the specimens were machined from trial components. The specimens in the rotor material were produced according to the following procedure [7, 19]: oxygen furnace melts were bottom poured for having a single ingot. The melts were vacuum-degassed (vacuum oxigen decarburization) using the lift-gas method to ensure low levels of hydrogen. A two step forging process was used to obtain the total deformation required to close pores in the disc centre. Finally, a lengthy cycled heat treatment in the annealing range was employed to reduce the levels of residual hydrogen. Material mechanical properties were enhanced by the heat treatments of an $840^{\circ} \mathrm{C}$ austenitization, salt bath quench and $600^{\circ} \mathrm{C}$ temper [20]. Finally, a prolongation of the rotor was cut for specimen machining.

The trial CRR was manufactured, by firstly performing hot forging by a press, in order to obtain uniform and fine grain size and to achieve the compression of microporosities. Forging temperature was $1100^{\circ} \mathrm{C}-1200^{\circ} \mathrm{C}$; afterwards a heat treatment at $1050^{\circ} \mathrm{C} \pm 5^{\circ} \mathrm{C}$ for 8 hours and with water cooling was applied. The forged ring was then machined, examined by non destructive tests and cold expanded (by the wedge method). Afterwards, a stress relief was applied at the constant temperature of $350^{\circ} \mathrm{C}$ for ten hours with cooling in calm air [20-21]. Finally, prolongations of the CRR were machined for specimen manufacturing. 
All the tests were performed on an 8032 INSTRON machine with the maximum capacity of 100 $\mathrm{kN}$. A novel loading-constraining device (Figure $1(\mathrm{C})$ ) was integrated to the existing clamps. It was developed to achieve an auto-compensation of the misalignments between upper and lower machine fixtures. The basic idea of such device (some details on its manufacturing, testing and validation in [22]) is to connect the threaded ends of each specimens to bushings: one of them is precision coupled to the machine slot at the lower clamp, while the other one is placed over a thrust bearing with sphered housing washers at the opposite clamp. One of the rings is mounted with opposite orientation: in this way the specimen end has two degrees of freedom, so that both lateral offsets and relative inclinations between the machine clamps can be compensated.

Both static and fatigue tests were performed (at ambient temperature): the first ones were executed in displacement controlled conditions at the constant speed of $5 \mu \mathrm{m} / \mathrm{s}$ (finally verifying that the limits on stress gradient, according to [23] were satisfied). LCF tests were performed in strain controlled conditions for conformal specimens (tangential direction), while the available space was not sufficient for the extensometer application on shorter specimens (radial direction). To carry out their testing, a particular procedure, exposed below, was set up. The basic idea was to perform a control on the relative displacement $u$ between the specimen heads. The relationship between the stress $\sigma$ and the strain $\varepsilon$ can be written as in Eq. (1), according to the Ramberg-Osgood model. Such relationship is valid both for a local and for a nominal state of stress/strain and in static conditions. $\mathrm{K}$ indicates the static plasticity coefficient and $\mathrm{n}$ the static hardening exponent, while $\mathrm{E}$ is the Young's modulus. A similar relationship is valid for a cyclic load, just replacing $\mathrm{K}$ by the cyclic plasticity coefficient $\mathrm{K}$ ' and $\mathrm{n}$ by the cyclic hardening exponent $\mathrm{n}$ '.

$$
\varepsilon=\frac{\sigma}{E}+\left(\frac{\sigma}{K}\right)^{\frac{1}{n}}
$$

Considering the nominal state of stress and an axial tension/compression load $\mathrm{N}$ ( $\mathrm{x}$ indicates the axial coordinate from the specimen centre towards the end, see also Figure 1 (B)):

$$
\sigma(x)=\frac{F}{A(x)}
$$

Equation (3) shows the well known relationship between the strain $\varepsilon$ and the displacement $\mathrm{u}$ :

$$
\varepsilon=\frac{d u}{d x} \Leftrightarrow d u=\varepsilon(x) d x \Leftrightarrow u=\int_{-l / 2}^{+l / 2} \varepsilon(x) d x=2 \int_{0}^{+l / 2}\left[\frac{\sigma(x)}{E}+\left(\frac{\sigma(x)}{K}\right)^{\frac{1}{n}}\right] d x
$$

By substituting Eq. (2) in Eq. (3), we have the following that can be easily integrated on the whole length of the hourglass shape 1, to determine the displacement $u$ (Eq. (4)).

$$
u=2 \int_{0}^{+l / 2}\left[\frac{F}{E A(x)}+\left(\frac{F}{K A(x)}\right)^{\frac{1}{n}}\right] d x=2 \frac{F}{E} \int_{0}^{l / 2} \frac{d x}{A(x)}+2\left(\frac{F}{K}\right)^{\frac{1}{n}} \int_{0}^{l / 2}\left(\frac{1}{A(x)}\right)^{\frac{1}{n}} d x=2 \frac{F}{E} \cdot V+2\left(\frac{F}{K}\right)^{\frac{1}{n}} \cdot Z
$$

The integral terms, $\mathrm{V}$ and $\mathrm{Z}$ depend on the specimen geometry and can be easily calculated (for a known value of $n$ ) for that sketched in Figure 1 (B), and anyway for any other geometry, as the described procedure can have a general application. The Eq. (5) shows that in the force F displacement $u$ dual domain a law that is very similar to Eq. (1) can be applied. 


$$
u=2 \frac{F}{E} \cdot V+\left(\frac{F 2^{n} Z^{n}}{K}\right)^{\frac{1}{n}}=\frac{F}{E_{u}}+\left(\frac{F}{K_{u}}\right)^{\frac{1}{n}}, E_{u}=\frac{E}{2 V}, K_{u}=\frac{K}{(2 Z)^{n}}
$$

On the basis of Eq. (5), with respect to the classical approach in the $\sigma-\varepsilon$ domain, it is equivalent to operate in the dual $\mathrm{F}-\mathrm{u}$ domain, by experimentally determining the static or the cyclic $\mathrm{F}-\mathrm{u}$ curve, with related parameters $\mathrm{E}_{\mathrm{u}}, \mathrm{K}_{\mathrm{u}}, \mathrm{n}$ or $\mathrm{K}_{\mathrm{u}}^{\prime}, \mathrm{n}$ '. Afterwards, by considering Eq. (5), the coefficients $\mathrm{E}, \mathrm{K}, \mathrm{K}$ ' (in the $\sigma-\varepsilon$ domain) can be easily computed, while the hardening exponent (n or n') remains the same in both domains. This conclusion appears to be particularly interesting, as it is much easier to operate in the $\mathrm{F}-\mathrm{u}$ domain, with control on displacement $\mathrm{u}$, rather than in the $\sigma-\varepsilon$ domain, requiring a strain control.

In the dual domain the fatigue tests were performed by controlling the relative displacements $u$ between the specimens ends: it could be easily achieved, by mounting circular bushings at the extremities, and by applying a conventional axial extensometer on them, as shown in Figure 2 (C).

A final interesting remark is that it can be experimental shown that in the dual domain there is also a homothetic relationship between the $\mathrm{F}-\mathrm{u}$ cyclic curve and the $\Delta \mathrm{F}-\Delta \mathrm{u}$ hysteresis cycles. Thus, by combining the following equations (Eq. (6)), also considering Eq. (2), it is possible to establish the relationship between the controlled displacement amplitude $\Delta \mathrm{u} / 2$ and the corresponding strain amplitude $\Delta \varepsilon / 2$ (at the specimen centre, for $\mathrm{x}=0$ and where $\Delta \sigma=\Delta \mathrm{F} /$ nominal section).

$$
\left\{\begin{array}{l}
\frac{\Delta \varepsilon}{2}=\frac{\Delta \sigma}{2 E}+\left(\frac{\Delta \sigma}{2 K^{\prime}}\right)^{\frac{1}{n^{\prime}}} \\
\frac{\Delta u}{2}=\frac{\Delta F}{2 E_{u}}+\left(\frac{\Delta F}{2 K_{u}^{\prime}}\right)^{\frac{1}{n^{\prime}}}
\end{array}\right.
$$

\section{Experimental tests and results}

The experimental campaign was focused on the determination of the static and cyclic curves, and of the fatigue ones. During the tests a three-channel acquisition was performed, by monitoring the transmitted load $\mathrm{F}$, the strain reading by the extensometer and the machine actuator displacement. The measurement chain was composed by the analogical output of the INSTRON machine, an SCB68 platform and a DAQ Card NI 6062E (National Instruments, Mopac, Expwy, Austin, TX, USA). Data acquisition and recording were assisted by LabView programs developed for this particular application: such programs had an essential role for the automatic evaluation of the stabilized cycle condition and for crack initiation detection. The cycle was supposed to be stabilized when maximum load values had a percentage variation under $0.15 \%$, while failure was determined after a maximum $10 \%$ drop from the previous condition of stabilized cycle. The typical cycle shape in failure conditions (as shown in [18]) was observed in the last cycles of the trials and non destructive tests (with fluorescent penetrants) were executed on the cracked specimens to verify the real cracking.

Figure 2 (A) shows the front panel of the LabView program, while Figure 2 (B,C) shows the testing conditions for conformal and shorter specimens with bushings connected to their ends.

The cyclic and fatigue curves are shown in Figures 3-4 for all the tested configurations. Each cyclic curve was determined as an envelope of terminal points of about 25 stabilized cycles. In the cases of the specimens machined along the radial direction, $\mathrm{F}-\mathrm{u}$ cyclic curves were initially determined, and consequently coefficients $\mathrm{K}_{\mathrm{u}}{ }_{\mathrm{u}}$ and $\mathrm{n}$ ' by data linear regression in the logarithmic scale. Afterwards, the $\sigma-\varepsilon$ curves and related coefficient $\mathrm{K}^{\prime}$ were determined on the basis of the previously described numerical procedure. The fatigue curves were determined by performing tests at 8-10 strain amplitude levels with three replications. The Manson-Coffin curve was traced, by 
operating the decomposition of the total strain amplitude in its elastic and plastic components: the elastic and plastic lines were then determined by linear regression in the logarithmic scale. The performed procedure led to the computation of the fatigue strength and fatigue ductility coefficients and of the fatigue exponents, characterizing the material LCF behaviour.

The last step consisted in the determination of the tolerance bands for the aforementioned lines and curves and, as a consequence, of the tolerance values to be applied to the cited coefficients. Such procedure is described in the following paragraph. Figures 3-4 show the bands related to the regression lines and to the Manson-Coffin curve, in addition experimental points are sketched, considering the total strain amplitude (larger ones) and the elastic and plastic contributions only (smaller points).

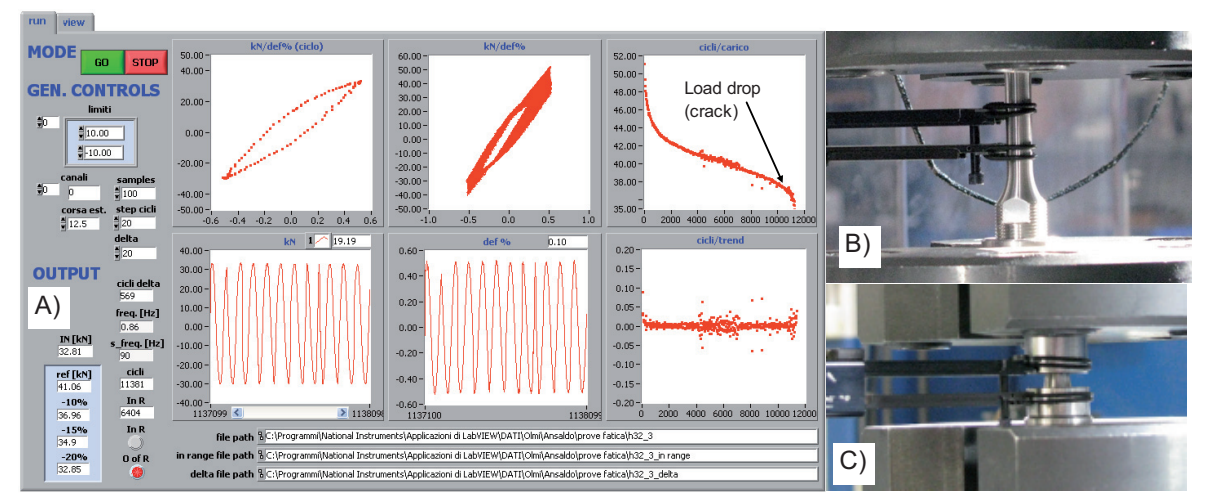

Fig. 2. LabView Front Panel (A) and experimental settings for conformal (B) and shorter specimens (C).
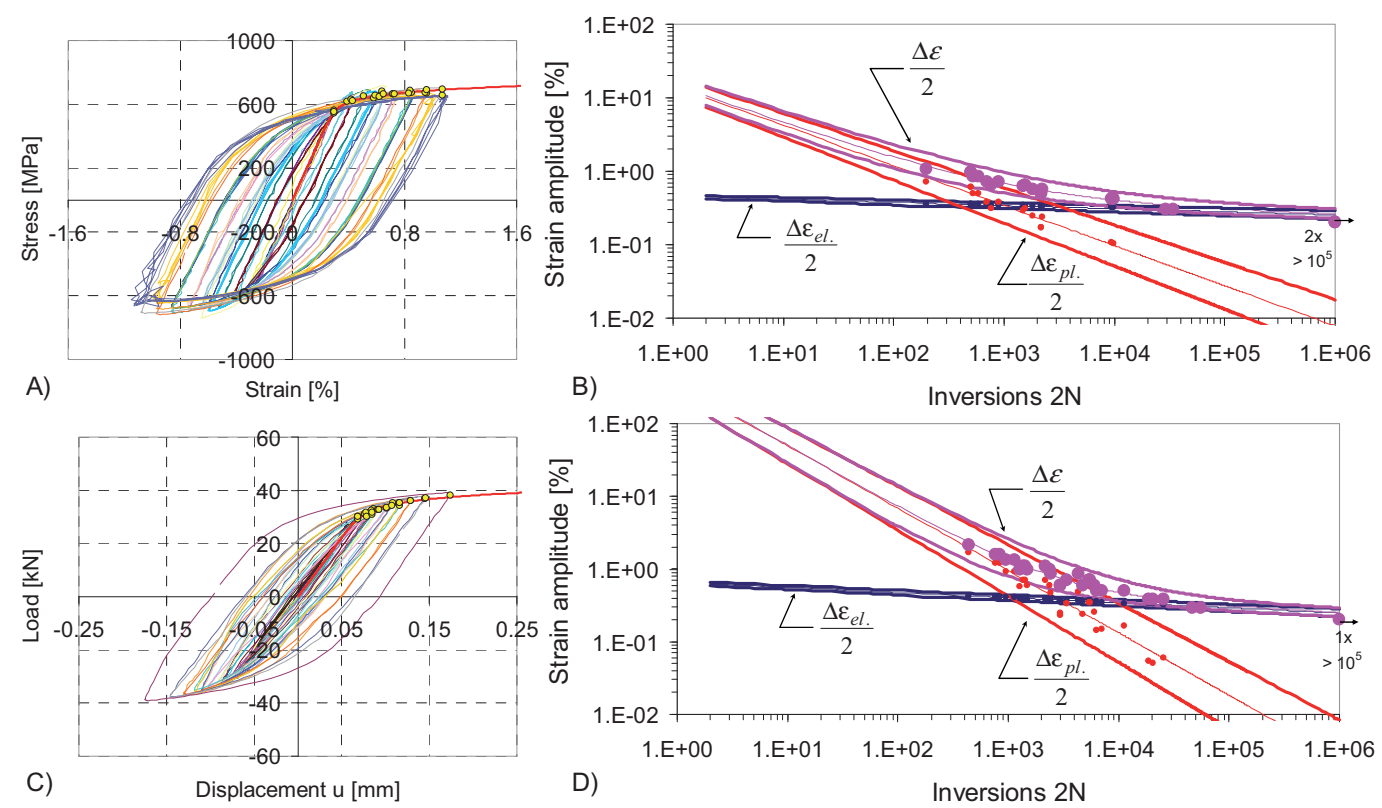

Fig. 3. Characterization of $26 \mathrm{NiCrMoV} 14$ 5: cyclic (A) and fatigue (B) curves in the tangential direction, cyclic (C) and fatigue (D) curves in the radial direction. 

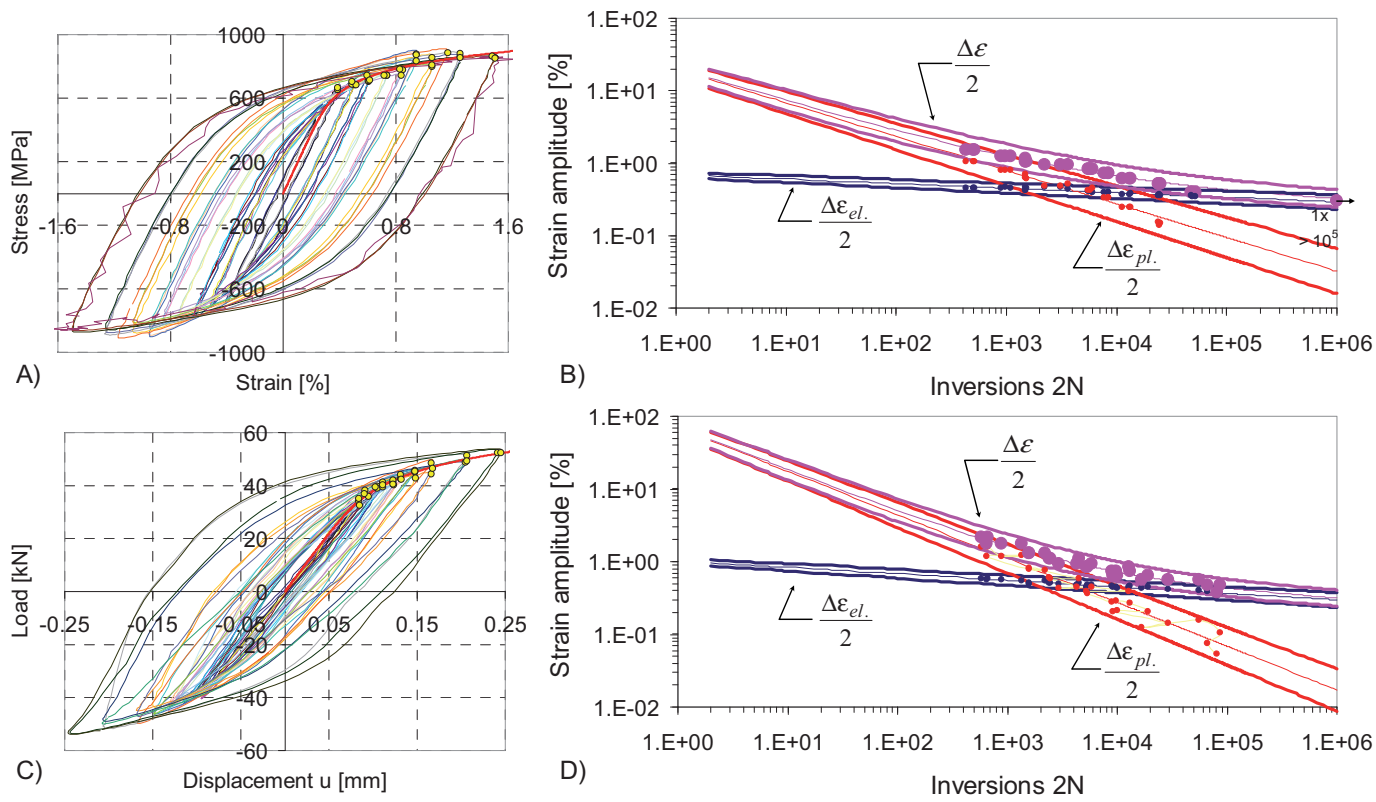

Fig. 4. Characterization of $18 \mathrm{Mn} 18 \mathrm{Cr}$ : cyclic (A) and fatigue (B) curves in the tangential direction, cyclic (C) and fatigue (D) curves in the radial direction.

\section{Discussion}

Both the materials involved in the experimentation have very good static properties: $26 \mathrm{NiCrMoV} 14$ 5 has a ultimate resistance of about $900 \mathrm{MPa}$ and a yield strength a bit lower than $800 \mathrm{MPa}$, while $18 \mathrm{Mn} 18 \mathrm{Cr}$ has both ultimate and yield strength higher than $1200 \mathrm{MPa}$. Like for high strength steels, cycling implies a softening effect, which can be observed in Figure 5. In the same Figure static and cyclic properties for the two materials are compared along the two machining directions. It can be noticed that the differences are negligible for the rotor material, while there is a gap lower than $10 \%$ (averaged value of about 5\%) at the highest strain values between the cyclic curves of the CRR material. Thus, not a strong anisotropy effect must be emphasized, regarding the static and cyclic behaviour.
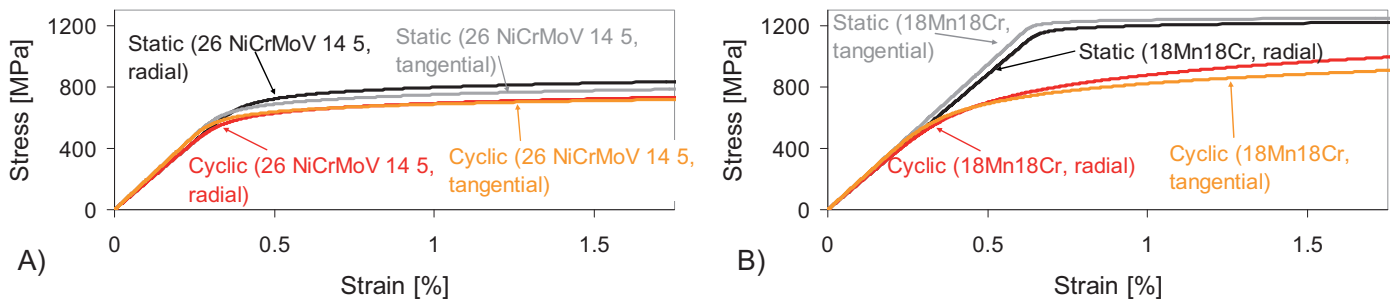

Fig. 5. Static and cyclic curves for $26 \mathrm{NiCrMoV} 145$ (A) and $18 \mathrm{Mn} 18 \mathrm{Cr}$ (B).

In the study of the LCF response, after determining the linear regressions in the elastic $\left(\Delta \varepsilon_{\mathrm{el}} / 2-\right.$ $2 \mathrm{~N})$ and in the plastic field $\left(\Delta \varepsilon_{\mathrm{pl}} / 2-2 \mathrm{~N}\right)$, the analysis was completed by discussing the sensitivity of the adopted models, i.e., by determining the error ranges of the regression tasks. For this purpose, the variance of the slope and of the constant term of each regression line were computed, according to [24], by estimating data point variance as the ratio between the quadratic sum of residuals (between the data points and the regression values) and the number of degrees of freedom. Minimum and 
maximum values for the slopes and the constant terms were then computed, by considering the worst scenario of twice the standard deviation scattering, and so that all the experimental points could be contained in the tolerance bands. The fatigue resistance coefficients could so be determined with few percentage points uncertainty, and with smalls scatterings for the other LCF parameters

Finally, to address the major purpose of this work, i.e. to discuss on the LCF behaviour depending on material properties and on the machining direction, the two-factor plan in Table 2 was analysed by the technique of the ANalysis Of VAriance (ANOVA). An original approach of this analysis was adopted to compare the elastic and plastic distributions, with error estimation as the quadratic sum of residuals. The result was that both the factors, material and machining direction, are significant at the 5\% significance level, without interaction. The material is the most significant: at the average $18 \mathrm{Mn} 18 \mathrm{Cr}$ LCF performance is $45 \%$ better than that of $26 \mathrm{NiCrMoV} 145$. As far as the anisotropy is concerned, the observation of Figure 6 makes it possible to allocate the differences between the fatigue curves in the two directions. It can be noticed that the response is slightly better in the radial direction (about 5\% improvement), mainly for the rotor material, even if such effect is evident only at the greatest strain amplitudes. On the other side, for low amplitude values, for $2 \mathrm{~N}$ ranging between $10^{4}$ and $10^{5}$ cycles (run-out value), the two curves (here represented without tolerance bands, for sake of clarity) are almost coincident, so that the impact of anisotropy appears to be negligible in this range of life. Such behaviour was observed for both materials, as confirmed also by the absence of interaction, as resulted by the performed analysis.
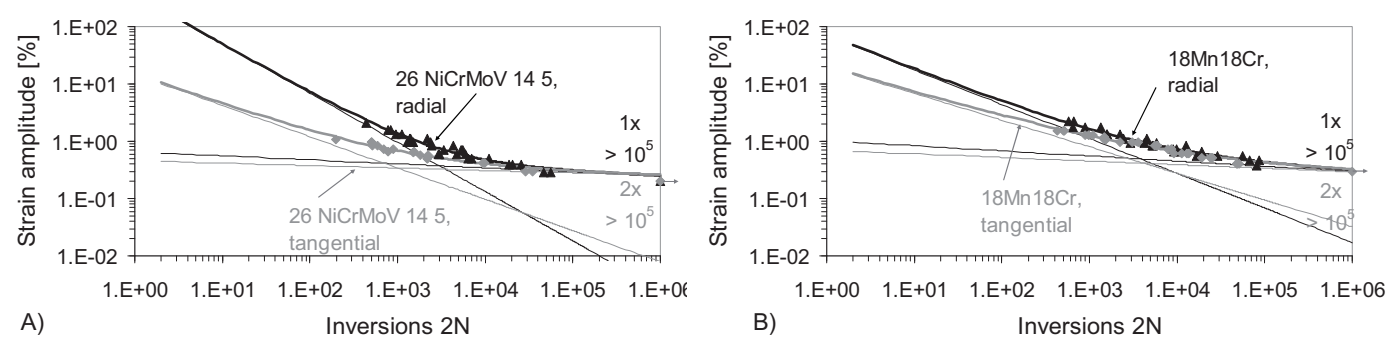

Fig. 6. Anisotropy effect on the Manson-Coffin curves for $26 \mathrm{NiCrMoV} 145$ (A) and $18 \mathrm{Mn} 18 \mathrm{Cr}$ (B).

\section{Conclusions}

The main purpose of this paper was to perform the characterization of two widely used steels for turbogenerator manufacturing, evaluating the anisotropy properties in the static, cyclic, fatigue behaviour. On the basis of the performed experimental activities, the following remarks can be made:

- The characterization task was carried out by following a two-factor plan, using specimens machined from trial rotor and CRR components along the tangential and the radial directions.

- A novel loading-constraining device was used for misalignment compensation, while an original methodology was adopted for performing strain controlled tests on very short specimens with not enough space available for axial extensometer application.

- Tests led to the determination of the static, cyclic and fatigue curves for each of the considered cases. A sensitivity analysis was performed on data scattering in the determination of regression lines, thus adding tolerance bands to the Manson-Coffin curves. A cyclic softening can be emphasized, greater for the CRR material, while the static and cyclic curves are not remarkably influenced by the machining direction (only between CRR material cyclic curves there is gap of some percentage points, just at the highest strains).

- The comparison between the fatigue curves showed that the material choice does have a strong influence at improving the LCF behaviour: fatigue performance is $45 \%$ increased for the CRR material. Anisotropy has a limited influence (performance about 5\% incremented in the radial 
direction), only at the greatest strain amplitudes, while at lower values, corresponding to a life between $10^{4}$ and $10^{5}$ inversions, closer to the real exercise conditions, such influence is negligible.

\section{References}

1. W.G. Moore, Evaluation, repair \& restoration of generator magnetic retaining rings, Proc. of the International Joint Power Generation Conference and Exhibition (1999).

2. W. Tong, Proc. of the 2004 ASME International Congress and Exposition, 167-171 (2004).

3. A. Balitskii, O. Krohmalny, I. Ripey, International Journal of Hydrogen Energy, 25, 167-171 (2000).

4. K. Orita, Y. Ikeda, T. Iwadate, J. Ishizaka, ISIJ Journal, 30, 587-593 (1990).

5. Y. Yamamoto, T. Namera, T. Otaka, H. Ito, M. Tari, Successful manufacturing and shop testing of the world's largest tandem 2-pole, 60Hz, 1,000MW turbine generator, Proc. of the IEEE Power Engineering Society Transmission and Distribution Conference, 1393-1398, (2001).

6. D.P. Peagno, Proc. of the Electrical Engineering Congress, 2, 251-258 (1994).

7. J.T. Murphy, R. Ballinger, H. Becker, D. Gwinn, S. Mathew, Proc. of the IEEE Power Engineering Society Transmission and Distribution Conference, 551-554 (1992).

8. W.Y. Maeng, D.D. Macdonald, Corrosion Science, 50, 2239-2250 (2008).

9. K.J. Nix, T.C. Lindley, Wear, 125, 1-2, 147-162 (1988).

10. V.P. Swaminathan, T.T. Shih, A. Saxena, Eng. Fracture Mechanics, 16, 6, 827-836 (1982).

11. S.A. Bashu, Journal of Testing and Evaluation, 18, 5, 363-368 (1990).

12. V.P. Swaminathan, H.G. Pennick, M.J. Jirinec, J. Madia, Remaining life assessment of the East River 7 high pressure rotor, Proc. of the International Joint Power Generation Conference (1991).

13. A.I. Balitskii, M. Diener, R. Magdowski, V.I. Pokhmurskii, M.O. Speidel, Anisotropy of fracture toughness of austenitic high nitrogen chromium-manganese steel, Proc. of the HNS (1998).

14. V.V. Skorchelletty, E.P. Silina, V.A. Zaytsev, V.V. Maslov, T.D. Lubeznova, Elektrychiskie stantsii, 12, 40-42 (1981).

15. M.O. Speidel, VGB Kraftwerkstechnik, 61, 5, 1048-1053 (1981).

16. N.L. Kilpatrick, M.I. Schneider, Proc. of the Third P900-Coloquium, 103-109 (1990).

17. A. Freddi, S. Curioni, G. Caligiana, La vita a fatica torsionale negli alberi dei turbo gruppi, (University of Bologna, Bologna, Italy, 1989).

18. ASTM E606-04, Standard Practice for Strain-Controlled Fatigue Testing (2004).

19. G. Klempner, I. Kerszenbaum, Operation and maintenance of large turbo generators (IEEE Press, New York, USA, 2004).

20. D. Bokelman, K. Forch, K.D. Haverkamp, Forging Technique for the Manufacture of a Heavy Flywheel with 108 t Forging Weight, Proc. of the 11th International Forgemasters Meeting (1991).

21. M.C. Sun, Y.H. Sun, R.K. Wang, Materials Letters, 58, 1340-1343 (2004).

22. G. Olmi, A. Freddi, Proc. of the XXXVIII AIAS National Conference, 1, 93, 1-14 (2009).

23. UNI EN 10002-1, Prova di trazione. Parte 1: Metodo di prova a temperatura ambiente (2004).

24. J.F. Doyle, Modern Experimental Stress Analysis (John Wiley and Sons Ltd., West Sussex, England, 2004). 